\title{
Extramedullary hematopoiesis: A report of two cases
}

\author{
HUAN-ZHU ZHANG, YING LI, XIN LIU, BAO-RONG CHEN, GUO-HUA YAO and YU-NA PENG \\ Oncology Department, The First Affiliated Hospital of Guangzhou Medical University, \\ Guangzhou, Guangdong 100044, P.R. China
}

Received August 6, 2015; Accepted August 11, 2016

DOI: $10.3892 /$ etm.2016.3855

\begin{abstract}
Extramedullary hematopoiesis (EMH) is defined as hematopoiesis occurring in organs outside of the bone marrow. The present report describes two cases of thalassemic patients with paraspinal medullary hematopoiesis and analyzes the clinical manifestations, imaging, pathology, diagnosis and treatment of EMH. In addition, a supplementary review of previously published cases is provided along with a review of the related literature. Computed tomography (CT) of the first case revealed multiple paraspinal masses, and the largest was $6.2 \times 8.0 \mathrm{~cm}$ in diameter. Likewise, CT of the second patient revealed multiple paraspinal masses in the bottom of the left thoracic cavity, and the largest was measured $10.1 \times 10.5 \mathrm{~cm}$. The two cases underwent surgical biopsy and the findings were compatible with a diagnosis of EMH. In conclusion, EMH is a compatible and rare disease, and should be distinguished from other neoplasms. EMH must considered when masses with characteristic radiologic appearance are detected in patients with thalassemia intermedia.
\end{abstract}

\section{Introduction}

Extramedullary hematopoiesis (EMH) refers to the hematopoiesis that occurs in organs other than bone marrow (1). It is a compensatory phenomenon that results in the production of blood cell precursors outside the marrow in patients with thalassemia intermedia. It has been reported to occur in $\sim 15 \%$ of cases of thalassemia, and it occurs in myelofibrosis and in other anemic conditions (2). The process can involve virtually any organ or tissue, such as the liver, spleen, abdominal viscera, pleura, lymph nodes, adrenal glands, breast, thymus, kidneys, gastrointestinal tract, intracranial structures and paraspinal regions (3-6). EMH is a rare entity and often asymptomatic but can sometimes lead to symptomatic tumor-like masses.

Correspondence to: Professor Huan-Zhu Zhang, Oncology Department, The First Affiliated Hospital of Guangzhou Medical University, 151 Yanjiang Road, Guangzhou, Guangdong 100044, P.R. China

E-mail: clara_0709@163.com

Key words: extramedullary hematopoisis, thalassemia, neoplasm, anemia, bone marrow
Treatment options are controversial and include hypertransfusion, surgical excision, radiotherapy, and hydroxyurea (7). Paraspinal involvement is the associated with morbidity secondary to spinal cord compression (8). Among the various body regions reported, paraspinal involvement deserves special attention due to the debilitating clinical consequences and challenges in its diagnosis and management. The present study described two cases with paraspinal medullary hematopoiesis in two thalassemic patients. Verbal informed consent was obtained from both patients.

\section{Case report}

Case 1. A 54-year-old male was admitted to the First Affiliated Hospital of Guangzhou Medical University (Guangzhou, China) in May 2012 with complaints of dizziness and hypodynamic condition for one month. The patient was known to have $\alpha$-thalassemia. On the day of admission, the patient's hemoglobin level was $71 \mathrm{~g} / 1$ (normal range, 120-160 g/l), the red blood cell count was $3.48 \times 10^{12}$ cells/l (normal range, $4.0-5.5 \times 10^{12}$ cells/l) and other routine hematological examinations and biochemical tests were within normal limits. Non-contrast and contrast-enhanced chest computed tomography (CT) scan revealed multiple paraspinal masses in the rear mediastinum, and the largest was measured $6.2 \times 8.0 \mathrm{~cm}$ (Fig. 1). The tumors were well-demarcated, and demonstrated heterogeneous density, ranging between 57 and 97 Hounsfield units. Considering the size of the mass and the possibility of multiple neurofibroma, a surgical biopsy was performed and showed infiltration by megakaryocytes, erythroblasts and myeloid cells (Fig. 2). Immunohistochemical staining was performed by obtaining paraspinal mass from the rear mediastinum, fixing in $10 \%$ paraformaldehyde for $6 \mathrm{~h}$ at room temperature and embedding it in paraffin. Sections $(4 \mu \mathrm{m})$ were then dewaxed in xylene, rehydrated and blocked using $3 \%$ hydrogen peroxide in methanol for $30 \mathrm{~min}$ at room temperature with $10 \%$ bovine serum albumin/Tris-buffered saline for $30 \mathrm{~min}$ at room temperature. Sections were then incubated with primary antibodies against cluster of differentiation (CD)61 (1:100; cat. no. MAB-0604; Maixin Biotech Co., Ltd., Fuzhou, China), MP0 (1:1,000; cat. no. A0398; Dako Medical Device Technology Service Co., Ltd., Shanghai, China), CD15 (1:100; cat. no. M3631; Dako Medical Device Technology Service Co., Ltd.), CD3 (1:50; cat. no. IR503; Dako Medical Device Technology Service Co., Ltd.), CD5 (1:100; cat. no. NCL-L-CD5-4CT; Quanhui Business Co., 


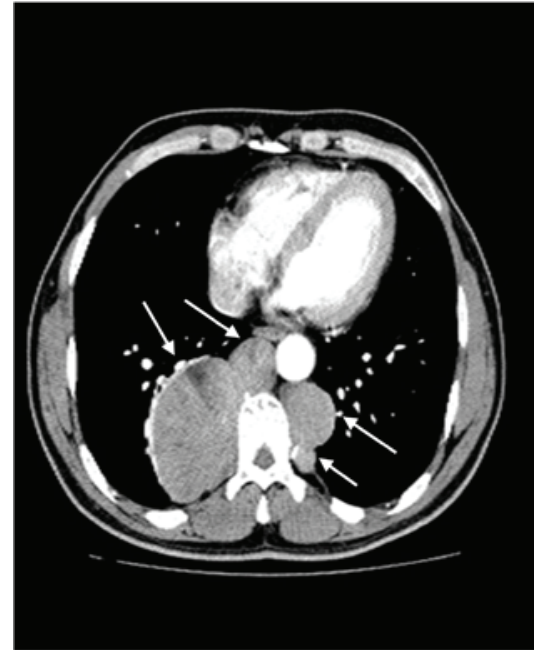

Figure 1. Multiple paraspinal masses (indicated with arrows) in case 1 in the rear mediastinum were observed, and the largest measured $6.2 \times 8.0 \mathrm{~cm}$.

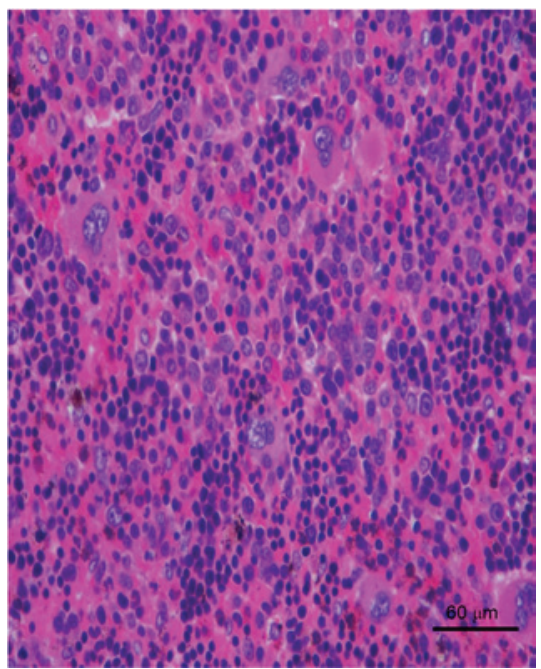

Figure 2. A biopsy from case 1 was showed infiltration with megakaryocytes, erythroblasts and myeloid cells.

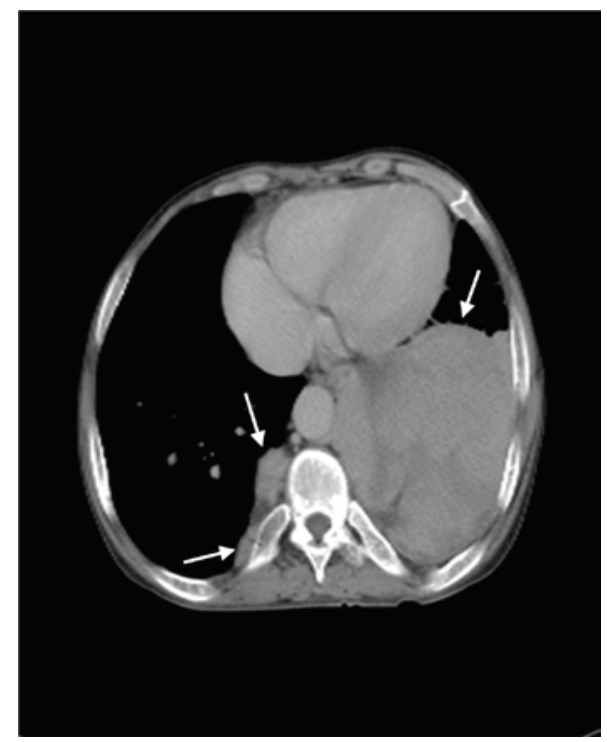

Figure 3. Multiple paraspinal masses in the bottom of the left thoracic cavity were observed in case 2 , and the largest measured $10.1 \times 10.5 \mathrm{~cm}$.

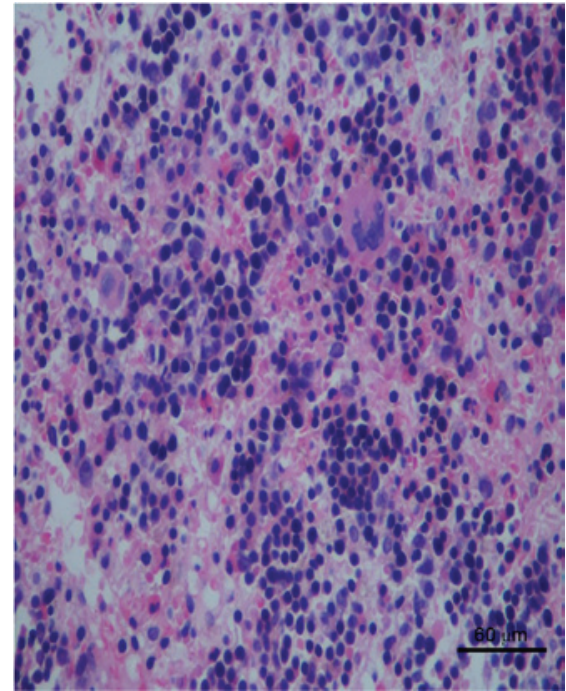

Figure 4. A biopsy from case 2 showed infiltration with megakaryocytes, erythroblasts and myeloid cells.

Ltd., Gaungzhou, China), CD20 (1:200; cat. no. IR604; Maixin Biotech Co.,Ltd.), CD79a (1:200; cat. no. IR621; Dako Medical Device Technology Service Co., Ltd.), CD138 (operating fluid; cat. no. IR642; Dako Medical Device Technology Service Co., Ltd.), LCA (1:100; cat. no. MAB-0037; Maixin Biotech Co., Ltd.) and CD117 (1:500; cat. no. A4502; Dako Medical Device Technology Service Co., Ltd.) at $37^{\circ} \mathrm{C}$ for $25-60 \mathrm{~min}$. Then, sections were then incubated with horseradish-peroxidase-conjugated goat and rabbit secondary antibodies. Slides were then counterstained with hematoxylin. Negative controls were performed by replacing the primary antibodies with phosphate-buffered saline.

Immunohistochemical analysis showed CD3/CD20/CD5/CD79a/CD138/CD68/leukocyte common antigen diffuse positive, $C D 117$ negative, $C D 15$ positive and myeloperoxidase $(M P O)$ positive corresponding to $E M H$. The patient experienced ablation of the biggest mass, and was free from recurrence after 2 years of follow-up.

Case 2. The second patient was a 48-year-old male, who presented with multiple masses in left thoracic cavity for 18 years and shortness of breath for 3 months. The patient was admitted to the First Affiliated Hospital of Guangzhou Medical University in December 2013, and was known to have severe $\beta$-thalassemia. The patient's hemoglobin level was $59 \mathrm{~g} / \mathrm{l}$ and the red blood cell count was $3.06 \times 10^{12} / 1$. CT scan revealed multiple paraspinal masses in the bottom of the left thoracic cavity, and the largest measured $10.1 \times 10.5 \mathrm{~cm}$ (Fig. 3). The tumors were well-demarcated and demonstrated heterogeneous density, ranging between 51 and 89 Hounsfield units. Considering the size of the mass and the possibility of multiple neurofibroma, a surgical biopsy was performed. Microscopically, the excised tumor exhibited characteristic megakaryocytes, erythroblasts and myeloid cells (Fig. 4). Immunohistochemical staining, performed as described above, showed that the tumor was positive for MPO, glycophorin and CD61, and diffuse positive for $\mathrm{CD} 20 / \mathrm{CD} 3$. The findings were compatible with a diagnosis of EMH. The patient was not provided any other treatment 
following a surgical biopsy, and was free from recurrence after 2 years of follow-up.

\section{Discussion}

EMH is defined as hematopoiesis occurring in organs outside of the bone marrow. It occurs if the bone marrow is no longer functional and is identified in a number of hematological diseases; its occurrence in chronic hemolytic anemias remains highest, particularly in transfusion-independent thalassemia intermedia (9-12). Ineffective red cell production by the bone marrow forces expansion of the hematopoietic tissue outside the marrow medulla and results in hematopoietic compensatory involvement, particularly in the form of masses, of other areas in the body (13).

Among the various body regions described, paraspinal involvement requires particular attention due to the debilitating clinical consequences and challenges in management and diagnosis. A paraspinal location in hematopoietic tissue occurs in $11-15 \%$ of cases with EMH (8). There is some predilection for the site of spinal cord involvement by the hematopoietic tissue. The thoracic region and, to a lesser extent, the lumbar region are the most frequently involved sites (14). The cause of this predilection is uncertain; however, these sites are understood to normally engage in active hematopoiesis in the fetus during gestation (14). This pathway typically stops at birth, but the extramedullary hematopoietic vascular connective tissues retain the ability to produce red cells under conditions of long-standing ineffective erythropoiesis (14).

Paraspinal EMH typically presents as pseudotumors, and can sometimes result in symptomatic tumor-like masses, which may cause a variety of neurological symptoms due to spinal compression. However, it is understood that $>80 \%$ of cases do not have signs and symptoms related directly to the disorder, and the lesions are typically discovered incidentally by radiologic techniques (15-17). The development of neurologic symptoms is suggested to depend on the chronicity of the disease, with neurologic symptoms most frequently being reported during the third and fourth decades of life (18), although few reports described presentation as early as the first decade of life (19-21). The male to female ratio reaches 5:1 (18). Various clinical presentations have been reported including back pain, lower extremity pain, parasthesia, abnormal proprioception, exaggerated or brisk deep tendon reflexes, Babinski response, Lasègue sign, paraparesis, paraplegia, ankle clonus, spastic gate, urgency of urination and bowel incontinence (22). The size and location of lesions and the extent of spinal cord involvement determines the severity, acuteness and multiplicity of signs and symptoms (22).

Although the history and physical examination may help narrow the differential diagnosis, radiographic imaging remains essential to confirm the existence of hematopoietic tissue. Characteristic appearance has been observed primarily using magnetic resonance imaging (MRI) or CT scan. MRI is the diagnostic investigation of choice. Paraspinal EMH appears as unique, multiple iso- or hyperintense masses, with homogeneous enhancement following contrast administration (23). These masses are usually lobular, well-circumscribed masses of intermediate signal intensity on T1-weighted images and low signal intensity on T2-weighted images $(23,24)$. The CT appearance is characterized by the heterogeneous soft tissue density mass. The diagnosis is confirmed following surgical removal of the mass. Biopsy remains the gold standard (25).

Because of its rarity, there is no standard treatment approach in patients with symptomatic EMH, and no evidence-based guidelines for the treatment of EMH. Therapy typically depends on the severity of symptoms, size of the mass, patient's clinical condition and previous treatment. Numerous treatment options have been described, including transfusion therapy, laminectomy, radiotherapy and the use of fetal hemoglobin, inducing agents that decrease the hematopoietic drive. However, the ideal management scheme remains controversial. Until large prospective trials evaluate the efficacy and safety of the available treatment options, both in single and in combination therapy, an individualized approach should be devised (26).

In conclusion, EMH is a rare disease, which is complicated to preoperatively distinguish from other neoplasms. In the present study, two patients with EMH are presented. The two patients underwent surgery and the pathological findings indicated that EMH was the correct diagnosis. These two cases suggest that EMH must be taken into consideration when masses with characteristic radiologic appearance are identified in patients with various hematological diseases, including thalassemia intermedia (4).

\section{Acknowledgements}

The authors thank the staff of the Radiology Department of the First Affiliated Hospital of Guangzhou Medical University, and Professor Xia Gu of the Pathology Department at The First Affiliated Hospital of Guangzhou Medical University (Guangzhou, China) for kindly providing the figures of the two patients.

\section{References}

1. Bozzini CE, Barrio Rendo ME, Devoto FC and Epper CE: Studies on medullary and extramedullary erythropoiesis in the adult mouse. Am J Physiol 219: 724-728, 1970.

2. Papavasiliou C: Clinical expressions of the expansion of the bone marrow in the chronic anemias: The role of radiotherapy. Int J Radiat Oncol Biol Phys 28: 605-612, 1994.

3. Sanei Taheri M, Birang SH, Shahnazi M and Hemadi H: Large splenic mass of extramedullary hematopoiesis. Iran J Radiol 2: 99-101, 2005.

4. Miyake H, Matsuda M, Iyomasa S and Mizuno K: Presacral extramedullary hematopoiesis. Surgery 135: 112-113, 2004.

5. Aessopos A, Tassiopoulos S, Farmakis D, Moyssakis I, Kati M, Polonifi K and Tsironi M: Extramedullary hematopoiesis related pleural effusion: The case of beta-thalassemia. Ann Thorac Surg 81: 2037-2043, 2006.

6. Al-Habib H and Hadzikaric N: Spinal cord compression due to intraspinal extramedullary hematopoiesis in thalassemia intermedia. Neurosciences (Riyadh) 12: 261-264, 2007.

7. Eskazan AE, Ar MC and BaslarZ: Intracranial extramedullary hematopoiesis in patients with thalassemia: A case report and review of the literature. Transfusion 52: 1715-1720, 2012.

8. Dore F, Cianciulli P, Rovasio S, Oggiano L, Bonfigli S, Murineddu M, Pardini S, Simonetti G, Gualdi G, Papa G, et al: Incidence and clinical study of ectopic erythropoiesis in adult patients with thalassemia intermedia. Ann Ital Med Int 7: 137-140, 1992.

9. Cromwell LD and Kerber C: Spinal cord compression by extramedullary hematopoiesis in myeloid metaplasia. Radiology 128: 118,1978 . 
10. de Morais JC, Spector N, Lavrado FP, Nobre LF, de Mattos JP, Pulcheri W, Nucci M, Novis S and de Oliveira HP: Spinal cord compression due to extramedullary hematopoiesis in the proliferative phase of polycythemia vera. Acta Haematol 96: 242-244, 1996.

11. Haran M and Ni S: Recurrent reversible paraplegia. Lancet 357: 1092,2001

12. Taher A, Isma'eel $\mathrm{H}$ and Cappellini MD: Thalassaemia intermedia: Revisited. Blood Cells Mol Dis 37: 12-20, 2006.

13. Shin KH, Sharma S, Gregoritch SJ, Lifeso RM, Bettigole R and Yoon SS: Combined radiotherapeutic and surgical management of a spinal cord compression by extramedullary hematopoiesis in a patient with hemoglobin E beta-thalassemia. Acta Haematol 91: 154-157, 1994.

14. Tsitouridis J, Stamos S, Hassapopoulou E, Tsitouridis K and Nikolopoulos P: Extramedullary paraspinal hematopoiesis in thalassemia: CT and MRI evaluation. Eur J Radiol 30: 33-38, 1999.

15. Parsa K and Oreizy A: Nonsurgical approach to paraparesis due to extramedullary hematopoiesis. Report of two cases. J Neurosurg 82: 657-660, 1995.

16. Richter E: Extramedullary hematopoiesis with intraspinal extension in thalassemia. Aktuelle Radiol 3: 320-322, 1993 (In German).

17. Dore F, Pardini S, Gaviano E, Longinotti M, Bonfigli S, Rovasio S, Tomiselli A and Cossu F: Recurrence of spinal cord compression from extramedullary hematopoiesis in thalassemia intermedia treated with low doses of radiotherapy. Am J Hematol 44: 148, 1993.

18. Salehi SA, Koski T and Ondra SL: Spinal cord compression in betathalassemia: Case report and review of the literature. Spinal Cord 42: 117-123, 2004.
19. Cardia E, Toscano S, La Rosa G, Zaccone C, d'Avella D and Tomasello F: Spinal cord compression in homozygous beta thalassemia intermedia. Pediatr Neurosurg 20: 186-189, 1994.

20. Chehal A, Aoun E, Koussa S, Skoury H, Koussa S and Taher A: Hypertransfusion: A successful method of treatment in thalassemia intermedia patients with spinal cord compression secondary to extramedullary hematopoiesis. Spine 28: E245-E249, 2003.

21. Ileri T, Azik F, Ertem M, Uysal Z and Gozdasoglu S: Extramedullary hematopoiesis with spinal cord compression in a child with thalassemia intermedia. J Pediatr Hematol Oncol 31: 681-683, 2009.

22. Haidar R, Mhaidli H and Tacher AT: Paraspinal extramedullary hematopoiesis in patients with thalassemia intermedia. Eur Spine J 19: 871-878, 2010

23. Haidar S, Ortiz-Neira C, Shroff M, Gilday D and Blaser S: Intracranial involvement in extramedullary hematopoiesis: Case report and review of the literature. Pediatr Radiol 35: 630-634, 2005.

24. Debard A, Demasles S, Camdessanché JP, Duband S, Mohammedi R and Antoine JC: Dural localization of extramedullary hematopoiesis. Report of a case. J Neurol 256: 837-838, 2009.

25. Tan TC, Tsao J and Cheung FC: Extramedullary haemopoiesis in thalassemia intermedia presenting as paraplegia. J Clin Neurosci 9: 721-725, 2002.

26. Haidar R, Mhaidli H and Taher AT: Paraspinal extramedullary hematopoiesis in patients with thalassemia intermedia. Eur Spine J 19: 871-878, 2010. 